\section{PLANIFICACIÓN Y REDISTRIBUCIÓN DE BENEFICIOS DEL DESARROLLO URBANO: LA CONTRIBUCIÓN COMPENSATORIA EN ROSARIO, ARGENTINA*}

Beatriz Cuenya ${ }^{\alpha}$

Eduardo González ${ }^{\beta}$

\section{Resumen}

La redistribución de los beneficios que otorgan las normas urbanas mediante instrumentos de planificación es uno de los objetivos que persiguen los gobiernos locales progresistas preocupados por regular el desarrollo de las ciudades con criterios de equidad y oportunidad. La llamada Contribución Compensatoria por beneficios extraordinarios en urbanizaciones y edificaciones, puesta en marcha en la ciudad de Rosario desde el 2000, apunta en esa dirección. El instrumento presenta un carácter pionero y novedoso en Argentina, lo que fundamenta el interés por su análisis. Este artículo se inicia con un breve marco de referencia sobre los antecedentes latinoamericanos en materia de

\section{PLANNING AND REDISTRIBUTION OF THE BENEFITS OF URBAN DEVELOPMENT: COMPENSATORY CONTRIBUTION IN ROSARIO, ARGENTINA*}

\author{
Beatriz Cuenya ${ }^{\alpha}$ \\ Eduardo González ${ }^{\beta}$
}

\section{Abstract}

The redistribution of benefits derived from urban regulations in the form of planning instruments is a goal sought by progressive local governments who focus on regulating the design of cities according to criteria of equity and opportunity. The so-called Compensatory Contribution, which is based on the redistribution of excess profits for the development of urban areas and buildings, implemented in the city of Rosario in 2000, is directly related to such an objective. The present paper is based on the analysis of this pioneering and unprecedented instrument in Argentina, which begins with a brief reference framework on the Latin American experience 
captura y redistribución de plusvalías asociadas a actuaciones públicas. Luego se describe el contexto en el que surge el instrumento, se examinan sus principales características y encuadre normativo, se exponen los desafíos enfrentados durante su implementación, y se presentan los resultados logrados y sus posibilidades de replicación. El estudio de base se realizó en el marco de una convocatoria auspiciada por el Lincoln Institute of Land Policy dirigida a registrar instrumentos notables de desarrollo urbano, con el propósito de difundir sus rasgos de originalidad, innovación y/o novedad contribuyendo a afrontar fuertes desafíos de la planificación urbana en la región.

\section{PALABRAS CLAVE: URBANISMO, POLÍTICAS} REDISTRIBUTIVAS, INSTRUMENTOS NOTABLES.

Recibido: 12/01/2016

Aceptado: 04/10/2016

* El estudio que dio origen a este artículo fue financiado por el Lincoln Institute of Land Policy (Programa para América Latina), bajo el título Compensatory Contribution for Extraordinary Urbanization and Building Benefits: Noteworhty Instrument of Urban Policy in Rosario, Argentina. Lincoln reference: LBC092013.

$\alpha \quad$ Consejo Nacional de Investigaciones Científicas y Técnicas (CONICET). Buenos Aires, Argentina (beatrizcuenya@gmail.com)

$\beta \quad$ Secretaría de Planeamiento Urbano de la Municipalidad de Rosario. Rosario, Argentina (eagz58@hotmail.com) in the generation and redistribution of capital gains associated with public actions. There follows a description of the context in which this instrument was conceived, an analysis of its main characteristics and regulatory framework, and an overview of the challenges faced by this program during its implementation phase. This paper also describes the results and the scope for replication of this instrument. The present study was conducted within the framework of a call for research proposals sponsored by the Lincoln Institute of Land Policy, which is intended to create a list of noteworthy urban development instruments in order to disseminate their original and innovating features, thus contributing to confronting urban planning challenges in the region.

\section{KEYWORDS: URBAN PLANNING, REDISTRIBUTIVE POLICIES, NOTEWORTHY INSTRUMENTS.}

Received: 12/01/2016

* This paper is based on a Lincoln Institute of Land Policy-funded project entitled "Compensatory Contribution for Extraordinary Urbanization and Building Benefits: Noteworhty Instrument of Urban Policy in Rosario, Argentina". Lincoln reference: LBC092013.

$\alpha \quad$ National Scientific and Technical Research Council (CONICET). Buenos Aires, Argentina (beatrizcuenya@gmail.com).

$\beta \quad$ Department of Urban Planning, Municipality of Rosario. Rosario, Argentina (eagz58@hotmail.com). 


\section{Introducción}

La redistribución de los beneficios que otorgan las normas urbanas mediante instrumentos de planificación es uno de los objetivos que persiguen los gobiernos locales progresistas preocupados por regular el desarrollo de las ciudades con criterios de equidad y oportunidad.

La posibilidad de lograr ese objetivo en la región se potenció a partir de la aprobación de reformas constitucionales y legales que apelan explícitamente a la movilización de los incrementos en el valor del suelo (plusvalías), que resultan no solo de obras públicas sino también de regulaciones urbanísticas, como son: la incorporación de suelo rural a la planta urbana, la modificación de la zonificación de usos del suelo y la autorización de un mayor aprovechamiento del suelo. Desafiando la tradición de apropiación privada de las plusvalías asociadas a este tipo de regulaciones, las reformas en la legislación se apoyan en la idea de que resulta lógico, conveniente y equitativo que el Estado, en representación de los intereses colectivos, rescate ese beneficio para ponerlo al servicio de la comunidad. Algunas legislaciones avanzan en el reconocimiento de la función social de la propiedad y distinguen claramente entre el derecho de propiedad del suelo y el de construcción (Rolnik, 2001; Sandroni, 2011; Smolka, 2013).

Se ubican en la vanguardia de las aludidas reformas, Colombia (Ley 388 /97, Ley Nacional de Desarrollo Territorial) y Brasil (Constitución de 1988 y Estatuto de la Ciudad de 2001). Pero también países como Uruguay, México, Ecuador, Bolivia y Venezuela comenzaron a recibir la influencia de la legislación colombiana y brasilera en sus propios cuerpos jurídico-urbanísticos. Las motivaciones que se encuentran por detrás de esta renovación van desde la intencionalidad de producir cambios profundos en la sociedad hasta la búsqueda de alternativas atractivas para el financiamiento de obras urbanas (Jaramillo, 2001). Para muchos analistas, la actualización de los instrumentos jurídicos y de planeamiento da cuenta de una nueva manera de abordar la gestión de la ciudad, que jerarquiza los intereses de la sociedad y desplaza la centralidad que históricamente ha tenido el derecho individual de propiedad del suelo en la política urbana latinoamericana (Alfonsín, 2008; Fernandes, 2006).

Argentina ha avanzado en esa dirección aunque de manera aún limitada, ya que el sistema legal nacional sigue regulando los derechos de propiedad desde una perspectiva extremadamente 
conservadora ${ }^{1}$. Un ejemplo contundente: la reciente reforma, actualización y unificación del Código Civil y Comercial de la Nación, ampliamente debatida en el país durante 2013 y aprobada en 2014, no incluyó la función social de la propiedad entre sus disposiciones y nuevos derechos. Y eso ocurrió pese a que tal principio había sido contemplado en el proyecto de reforma del código (elaborado por una comisión bicameral con participación de la Corte Suprema de Justicia) y pese a que había sido fuertemente apoyado por las organizaciones sociales que impulsan la reforma urbana.

En ese escenario, la ciudad de Rosario aparece liderando en el país un proceso de renovación en sus instrumentos de planeamiento y de gestión urbana. Aquí se inscribe la llamada Contribución Compensatoria por beneficios extraordinarios en urbanizaciones y edificaciones, puesta en marcha desde la década del 2000. Su carácter pionero y novedoso ha fundamentado el interés por su análisis y difusión.

El trabajo que acá se presenta se realizó en el marco de una iniciativa que desde 2013 auspicia el Lincoln Institute of Land Policy (Programa para América Latina) dirigida a registrar instrumentos notables de desarrollo urbano, con la idea de contribuir a afrontar los fuertes desafíos de la planificación

1 Por sistema legal nacional se alude a la Constitución Nacional, el Código Civil y la interpretación que se hace de estos textos legales cuando se aplica la ley y se formulan las políticas urbanas. urbana en la región. Uno de esos desafíos consiste precisamente en dirimir si la urbanización puede orientar el crecimiento económico hacia el bienestar de la población o por si el contrario debe aceptar que la mayoría de la población permanezca excluida de los beneficios que genera y ofrece la ciudad. Bajo esa impronta, el carácter de notable de los instrumentos refiere a sus rasgos de originalidad, innovación y/o novedad. Pero también a aspectos como la relevancia del problema social que abordan, la singularidad en sus procedimientos de gestión, la habilidad de expandir su alcance jurídico original o la potencialidad de ser replicados en una escala mayor (Smolka y Furtado, 2014).

Desde nuestro punto de vista, el carácter notable del instrumento que se analiza tiene que ver no solo con su originalidad y novedad en el territorio argentino, como se dijo, sino también con la audacia de la reformulación normativa que lo precedió. Efectivamente, mediante una reforma del Código Urbano se bajaron drásticamente las alturas de edificación libremente permitidas hasta entonces en ciertas zonas de la ciudad, con lo cual, toda superación de ese nuevo límite pasó a convertirse en un beneficio extraordinario otorgable por el sector público mediante el pago en una compensación. En el contexto de fuertes inversiones inmobiliarias 
privadas, como se expone más adelante, la aplicación del instrumento abrió una oportunidad al municipio para fortalecer su capacidad recaudatoria y aplicar los recursos recolectados con un sentido redistributivo, ya que estos fueron derivados a la preservación urbanística y a programas de suelo y vivienda. Otro rasgo destacable del instrumento es la participación democrática que acompañó su puesta en práctica. La misma consistió en un ciclo de intensos debates, consultas a profesionales, audiencia pública e instancias de concertación entre diferentes actores involucrados, lo que le dio un alto grado de aceptación al instrumento. Por último, la aplicación exitosa del instrumento sirve para ilustrar que es posible desde la política urbana local sortear el conservadurismo del marco jurídico nacional, con respecto a la gestión del suelo urbano.

Luego de esta fundamentación introductoria del caso de estudio, se presenta un breve marco de referencia sobre los antecedentes latinoamericanos en materia de captura y redistribución de plusvalías asociadas a actuaciones públicas. Acá se destaca la experiencia de San Pablo, como el precedente más semejante a la Contribución Compensatoria de Rosario. A continuación, se analiza específicamente la Contribución: se describe el contexto en el que surge el instrumento, se examinan sus principales características y encuadre normativo, se exponen los desafíos enfrentados durante su implementación, y se presentan los resultados logrados y sus posibilidades de replicación.

\section{Captura y redistribución de beneficios del desarrollo urbano: antecedentes latinoamericanos}

Es sabido que en América latina existen instrumentos de larga data que permiten al sector público capturar las valorizaciones inmobiliarias producto de inversiones en obras públicas. El más difundido es la Contribución por Mejoras, que se usa precisamente para financiar las obras. Se basa en el principio de que si el Estado confiere a un individuo una ventaja particular mensurable es justo que deba pagar por ella. Según diversos estudios, la Contribución por Mejoras ha tenido una amplia aceptación, ya sea porque permitió al Estado afrontar las crecientes necesidades derivadas del proceso de urbanización en contextos de restricciones fiscales; ya sea porque resultó un mecanismo atractivo de recaudación extra presupuestaria fácil de ser aceptado por los propietarios, dada su directa vinculación con una obra concreta que los beneficia; o incluso porque contó con el apoyo de las empresas privadas de construcción 
especializadas en obras públicas que, en varios países, tienen sólida base empresarial y fuerte capacidad de lobby político ${ }^{2}$.

En contraste, los instrumentos destinados a capturar y redistribuir la valorización del suelo generada por decisiones administrativas del Estado (la normativa urbanística), no han sido tradicionalmente aplicados en la política urbana de la región. Las normas de tipo universal que regulan el fraccionamiento del suelo y la incorporación de suelo urbano, por lo general, no han sido aplicadas con el criterio de recuperar plusvalías. Muy por el contrario, como ilustra la literatura especializada, la historia de la urbanización latinoamericana está plagada de casos de no recuperación. Ello se atribuye básicamente al papel preponderante asignado a la propiedad privada en el desarrollo socioeconómico y cultural de las sociedades latinoamericanas, como ocurrió en la Argentina. No obstante ello, desde fines de la década de los 80 en adelante, como se mencionó antes, las reformas legislativas introducidas de forma pionera en Colombia y Brasil habilitaron y promovieron la aplicación de instrumentos que permiten al Estado captar una parte de los beneficios generados por las actuaciones urbanísticas

2 En Brasil, por ejemplo, la Contribuçao de Melhorias fue apoyada por las empresas de obras públicas que se consolidaron a partir del año 1930, a través de seminarios, debates técnicos y el apoyo político que proporcionaba en el Congreso nacional, la llamada bancada rodoviaria (bancada pro rutas y caminos), (Furtado, 1997). que regulan el uso del suelo urbano y del espacio aéreo urbano, y eventualmente redistribuirlos con propósitos de equidad y utilidad pública.

La gama de instrumentos de todo tipo actualmente disponibles en América Latina para captura de plusvalías fueron registrados, categorizados y examinados por Martim Smolka en un interesante informe que publicó recientemente el Lincoln Institute of Land Policy (Smolka, 2013). Tomando como referencia las categorías propuestas en este estudio, la Contribución Compensatoria de Rosario puede ubicarse en la categoría genéricamente llamada cargas por derechos de construcción. Los instrumentos incluidos en este grupo se basan en la separación entre los derechos de construcción y los derechos de propiedad del suelo, lo que permite al sector público recuperar (y redistribuir) los incrementos en el valor del suelo que resultan del desarrollo de derechos, más allá de una determinada línea de base establecida.

Dentro de la categoría de cargas por derechos de construcción, el instrumento más parecido a la Contribución Compensatoria de Rosario es la Contribución Onerosa por el Derecho Adicional de Construcción (OOCD por sus siglas en 
portugués) establecido en Brasil sobre la base de la Ley de 2001. La OOCD está basada en el siguiente principio: el derecho de construcción del propietario del suelo está limitado a un coeficiente básico, diferente del máximo que puede soportar el área. La OOCD impone una carga por el derecho a construir por encima de ese básico definido por el municipio, y hasta un nivel máximo establecido en el plan maestro. La legitimidad de esta carga está basada en dos principales ideas, según Smolka. La primera es la implícita aceptación de que para soportar mayores volúmenes de construcción o usos jerárquicos del suelo, se requieren mayores inversiones públicas en obras de infraestructura y servicios. La segunda alude al reconocimiento de que los metros cuadrados adicionales de construcción son considerados un patrimonio público que no puede otorgarse a algunos ciudadanos en detrimento de otros, a menos que se compense a la sociedad por obtenerlos.

De acuerdo a un estudio realizado por Sandroni (2011), para la aplicación de la OODC en San Pablo se establecieron coeficientes mínimos, básicos y máximos de uso del suelo, y se limitó la oferta de área edificable $e^{3}$. Las cargas asociadas con la OODC son impuestas en base a la diferencia entre el coeficiente máximo y básico de un lote. En algunas áreas urbanas, estas nuevas regulaciones redujeron los derechos de construcción, estableciendo un coeficiente básico de 1 para tierra que había sido designada 2 o más en la legislación previa. Paralelamente, en otras áreas, el municipio utilizó el OODC para extender el máximo coeficiente hasta 4 , en tierra que anteriormente podría desarrollarse hasta solamente 1 o 2. Se fijaron excepciones o reducciones en el coeficiente, lo que significa que no hay que pagar contribución por derechos adicionales de construcción para desarrollar vivienda social, hospitales, escuelas, espacios culturales u otras actividades de interés sociales. Según el citado autor, estas herramientas, utilizadas juntas, habilitaron al municipio a mejorar la eficiencia en la gestión del suelo, promover resultados socialmente positivos y aumentar los ingresos. En un lapso de cinco años los ingresos captados alcanzaron los 325 millones de dólares, que fueron depositados

3 El coeficiente mínimo se refiere a la utilización mínima establecida en el plan para que una propiedad cumpla con su función social; el coeficiente básico establece la superficie edificable que cualquier propietario tiene el derecho a desarrollar en virtud de su derecho de propiedad; y el máximo es la cantidad de desarrollo que podría ser respaldado por la infraestructura existente y los reglamentos de zonificación. La oferta potencial de construcción residencial y no residencial fue limitada en todos los distritos, excepto dentro del perímetro de 13 operaciones urbanas. 
en un fondo de desarrollo urbano ${ }^{4}$. Hacia 2008, los proyectos aprobados para ser financiados con este fondo incluían parques, drenaje y saneamiento, equipamiento comunitario, regularización de asentamientos irregulares y restauración de edificios de patrimonio histórico. Un riesgo importante señalado en el uso de la herramienta es que la motivación para aumentar los ingresos municipales pase por encima de los criterios de planeamiento establecidos en el Plan Maestro, llevando a autorizar mayor potencial constructivo cuando este se ha agotado, bajo la presión de los desarrolladores.

\section{Contexto de surgimiento de la Contribución Compensatoria: boom inmobiliario en Rosario}

La ciudad de Rosario (1,2 millones de habitantes en 2010) es la más importante de la provincia de Santa Fe, sin ser su capital y forma parte de la tercera área metropolitana del sistema urbano nacional.

4 Se exceptúan de este monto las 13 operaciones urbanas (Sandroni, 2011).
Desde comienzos de la década de los 2000 Rosario vive un período de notable esplendor urbano, basado en la confluencia de fuertes inversiones públicas y privadas, gestionadas por un gobierno socialista que exhibe casi 24 años de continuidad en la Intendencia. Durante estos años no solo se reconfiguró el paisaje físico y funcional de la ciudad sino que también se actualizaron sus instrumentos de planeamiento urbano con el objetivo, entre otros, de regular y orientar las ganancias extraordinarias emergentes del boom de la construcción ${ }^{5}$. Rosario se convirtió así en una ciudad pionera en la aprobación de instrumentos de captura y redistribución de plusvalías urbanas generadas por regulaciones urbanísticas. El instrumento que acá se analiza (en adelante Contribución Compensatoria) es un ejemplo que forma parte de este conjunto de cambios.

Superada la crisis económica nacional 2001/2002, la ciudad renovó su fisonomía urbana y edilicia en varios órdenes: mejora y creación de nuevos parques; incorporación de equipamientos culturales, deportivos y hospitalarios; construcción de

5 Por ejemplo, las inversiones privadas realizadas en la operación de renovación urbana Scalabrini Ortiz-Puerto Norte superan los 300 millones de dólares (sin incluir el valor del suelo) según la siguiente distribución: Alto Rosario Shopping USD \$ 50.000.000; Forum (Grupo TGLT) USD \$ 75.700.000; Ciudad Ribera (Ingeconser SA) USD\$ 56.600.000; Dolfines, Torre Embarcadero y NorthLink (Lattuca y Asoc.) 87.400.000; Maui-1era. Etapa (Grupo SerPort SA) USD\$38.200.000. La llamada Manzana 407 con grupos inversores locales varios alcanzan unos USD $\$ 62.000 .000$. 
avenidas y vías de acceso; montaje de grandes centros comerciales y proliferación de nuevos bares, restaurantes y comercios en el centro y frente a la costa; rehabilitación de edificios de valor patrimonial que refuerzan la revalorización del centro, y puesta en marcha de grandes operaciones de renovación urbana, como la creación de una nueva centralidad en Puerto Norte, en la que se alojan usos de alta gama con la participación de inversores y usuarios de altos ingresos (Levin, 2011).

En la base de esta importante renovación urbanística se ubica una mutación en su estructura económica. Sin abandonar sus actividades portuarias e industriales, la ciudad comenzó a consolidar su perfil como centro de servicios en el cual se fueron expandiendo los servicios ligados a las empresas culturales, exposiciones y eventos, y al turismo de alcance internacional. De manera complementaria, se registró la afluencia de capital inmobiliario, motorizado tanto por desarrolladores internacionales como por actores económicos locales beneficiarios de la política económica nacional impulsada desde 2003; estos últimos incluyen sectores ligados al campo y a la industria, así como profesionales independientes que demandan propiedades de alta gama para vivir, pero también como alternativa de inversión y de ahorro a futuro (Cuenya, González, Mosto y Pupareli, 2012).

Con el objetivo de planificar esa gran transformación económica, funcional y física de la ciudad, garantizando una redistribución equitativa de los costos y beneficios del desarrollo urbano, se abrió un ciclo de intensos debates, consultas a profesionales, audiencia pública e instancias de concertación, que se iniciaron en 2004 y culminaron en 2011 con la aprobación de un nuevo Código Urbano y un nuevo Plan Urbano Rosario 2007-2017.

El consenso logrado fue que el anterior Código Urbano de 1967, al haberse pensado para una ciudad que supuestamente tendría dos veces y media la cantidad de habitantes que alcanzó al cabo de tres décadas, definió un régimen de altura y densidades de edificación que no logró concretarse, ni por la demanda ni por la capacidad económica para materializarlo. En consecuencia, el nuevo código introduce tres nuevos cambios importantes: a) reduce rotundamente la altura permitida en algunas zonas donde hasta entonces se podía construir hacia arriba de manera ilimitada según lo que permitiera la tecnología constructiva (por ejemplo en la zona céntrica donde además predominan muchos edificios de valor patrimonial); b) preserva la altura en otros tramos (la mayoría de los barrios residenciales) y c) estimula mayores alturas en zonas de previsible expansión y en grandes corredores como avenidas o accesos a la ciudad.

Por su parte, el Plan Urbano 2007-2017 reconoce que la reformulación normativa introducida por el nuevo Código Urbano genera inequidades entre los propietarios del suelo, según las particularidades 
de cada zona, lo cual debe ser contrarrestado por el sector público a través de las herramientas del urbanismo. Contempla entonces el pago de compensaciones por parte de propietarios beneficiados por las normas. Prevé asignar los recursos obtenidos a obras públicas, subsidios a los propietarios de inmuebles catalogados para preservación y desarrollo de obras en otros barrios. A diferencia del anterior Plan Regulador de 1967, al que reemplaza, el nuevo Plan se caracteriza por su carácter inductivo antes que normativo, su fuerte acento en la gestión público-privada antes que regulador de la actividad privada, la incorporación de mecanismos de participación ciudadana, y la inclusión de un abanico de nuevos instrumentos que innovan en la práctica de la planificación y gestión de la ciudad.

\section{La Contribución Compensato- ria y su operatoria}

El concepto y la definición más general de la Contribución Compensatoria está incluido en el nuevo Plan Urbano Rosario 2007-2017 que establece lo siguiente:

Cuando -como producto de los cambios normativos introducidos (cambios de usos, alturas, factores de edificabilidad u otra modificación normativa realizada)- se verifica el otorgamiento de un "mayor aprovechamiento urbanístico" se establece el pago de un "precio compensatorio", el cual se destina en forma directa o indirecta a la realización de obras y/o compra de tierras (Plan urbano Rosario, 2011, p. 139).

Desde el punto de vista jurídico se trata de una exacción, una imposición, una exigencia del Estado local. Dado que no hay legislación argentina de nivel nacional que contemple algún cobro por la valorización resultante de la normativa urbanística (tampoco la Constitución lo prevé) la Contribución Compensatoria se encuadra en el Derecho Tributario. Si bien la Contribución Compensatoria no es asimilable a una tasa (ya que en el derecho tributario argentino las tasas se cobran por la prestación de un servicio) sí podría asemejarse a un derecho o una licencia que se aplican cuando el Estado otorga una autorización para realizar alguna acción regulada (licencia para conducir vehículos, para habilitar un comercio, etc.). En este caso se asemejaría a una licencia que autoriza al "constructor" a construir más superficie.

El primer sector de la ciudad donde se aplicó la Contribución Compensatoria, permitiendo comprender su lógica, es en un área céntrica de unas 10 cuadras, en lotes frentistas. En esta zona se limitó la altura a 36 metros, lo que supone una notable reducción de la altura ilimitada anteriormente permitida. A partir de esta nueva regla, aquellos propietarios que quieran hacer uso de una mayor altura por encima de los $36 \mathrm{~m}$ y hasta un 
máximo de $60 \mathrm{~m}$ deben pagar una Contribución Compensatoria.

La operatoria de este instrumento está desarrollada en un conjunto de ordenanzas que, a lo largo de casi una década (2004- 2013), fueron ampliando el ámbito de aplicación de la Contribución Compensatoria, desde un sector del área central hasta abarcar toda la ciudad. Las ordenanzas también fueron ajustando el monto económico de la compensación, así como la proporción de su asignación a los distintos fondos específicos.

Como norma general, la Contribución Compensatoria rige para aquellas zonas y parcelas donde el nuevo Código admite superación de la altura con respecto al área circundante (edificios de perímetro libre, parcelas frentistas o corredores urbanos, y tramos de completamiento entre edificios pre existentes de mayor altura). Como norma particular, también rige para grandes parcelas (más de $5000 \mathrm{~m}^{2}$ ) y edificios especiales (entre 1000 y 5000 $\left.\mathrm{m}^{2}\right)$.

El monto de la Compensación no tiene correspondencia necesaria con la valorización facultada, ya que se establece en base a un valor del $\mathrm{m}^{2}$ que varía según las zonas de la ciudad (área central, frente ribereño, anillos y cordones perimetrales). Se contemplan diversas modalidades de pago de la Contribución Compensatoria: aporte monetario; ejecución de obras públicas y donación de tierras. El destino de los fondos también es variado: fondo de tierras, fondo de obras públicas, fondo de preservación del patrimonio, fondo para la construcción de vivienda para sectores medios y obras directas ejecutadas por los urbanizadores o compra de tierra para vivienda social.

Las diversas ordenanzas que regulan la Contribución Compensatoria se resumen a continuación.

- Ordenanza 7799/2004 recalificación normativa para los lotes frentistas a las Avenidas de la Libertad y del Huerto. Autoriza a los propietarios de un área céntrica de unas 10 cuadras (av. de la Libertad y del Huerto) a hacer uso de un mayor aprovechamiento por incremento en altura (por encima de $36 \mathrm{~m}$ ) mediante el pago de una contribución compensatoria. Esta se calcula utilizando un precio base por $\mathrm{m}^{2}$ (actualizado anualmente) sobre la base de tasaciones de mercado realizadas por un organismo público especializado. Sobre dicho monto se aplica un porcentaje del $10 \%$. Los recursos se destinan al Fondo de Preservación Urbanística (50\%) y a la pavimentación de barrios periféricos (50\%).

- Ordenanza 8125/2006 plan especial Barrio Pichincha. Extiende lo establecido en la ordenanza anterior a las parcelas frentistas a parques en el Barrio Pichincha.

- Ordenanza 8243/2008 reordenamiento urbanístico del área central. Amplia el área de aplicación de la Contribución Compensatoria a 306 manzanas del área central (aproximadamente 
1200 parcelas) en un fragmento en el cual se estimula la renovación. Se autoriza aplicar el instrumento a partir de los $23 \mathrm{~m}$ de altura y se cambia el destino del 50\% de los fondos recaudados: en lugar de pavimentación de barrios periféricos pasan al Fondo Municipal de Obras Públicas.

- Ordenanza 8244/2008 reordenamiento urbanístico del primer anillo perimetral al área central. Extiende el ámbito de aplicación del instrumento al primer anillo perimetral al área central que comprende unas 360 manzanas. Se aplica en los lotes frentistas (o corredores urbanos) y los proyectos en Grandes Parcelas $\left(1500 \mathrm{~m}^{2} \mathrm{o}\right.$ más). No autoriza alturas por encima de las del área circundante y solo excepcionalmente pueden alcanzarse alturas mayores.

- Ordenanza 8692/2010 régimen diferencial para el desarrollo de proyectos edilicios especiales. Regula la aplicación de la Contribución Compensatoria, "en parcelas o grupos de parcelas que incluyan operaciones de renovación, rehabilitación y/o sustitución edilicia donde la Municipalidad considere pertinente la definición de indicadores de altura máxima y parámetros de distancias diferenciados de los genéricamente establecidos por la normativa vigente" y en virtud de la mayor superficie a construir resultante de la recalificación normativa. Divide en tres partes la asignación de la Contribución:
34\% para vivienda para sectores medios y el resto en partes iguales a patrimonio y obras públicas.

- Ordenanza 8980/2012 reordenamiento urbanístico del segundo anillo perimetral de la ciudad De Rosario. Extiende el ámbito de aplicación de la Contribución al Segundo Anillo Perimetral que comprende 1810 manzanas. Se aplica en los lotes frentistas y grandes parcelas.

- Ordenanza 9068/2013 difunde el ámbito de aplicación al resto de la ciudad.

- Ordenanza 9104 y 9105/2013 proyectos de articulación. Precisa para casos de linderos con distintas alturas y condicionantes, la posibilidad de articular morfológicamente con las construcciones vecinas, aplicándoseles la CC al superar determinadas alturas de tejido.

\section{Encuadre legal de la Contribución Compensatoria}

En la República Argentina, la mayoría de los municipios están facultados para confeccionar su respectivo expediente urbano y plan regulador, los que contienen las previsiones necesarias para la organización y control del desarrollo de la ciudad. Estas atribuciones les son "delegadas" por 


\section{FIGURA 1. ZONAS DE APLICACIÓN DE LAS NORMAS URBANÍSTICAS EN LA CIUDAD DE ROSARIO}

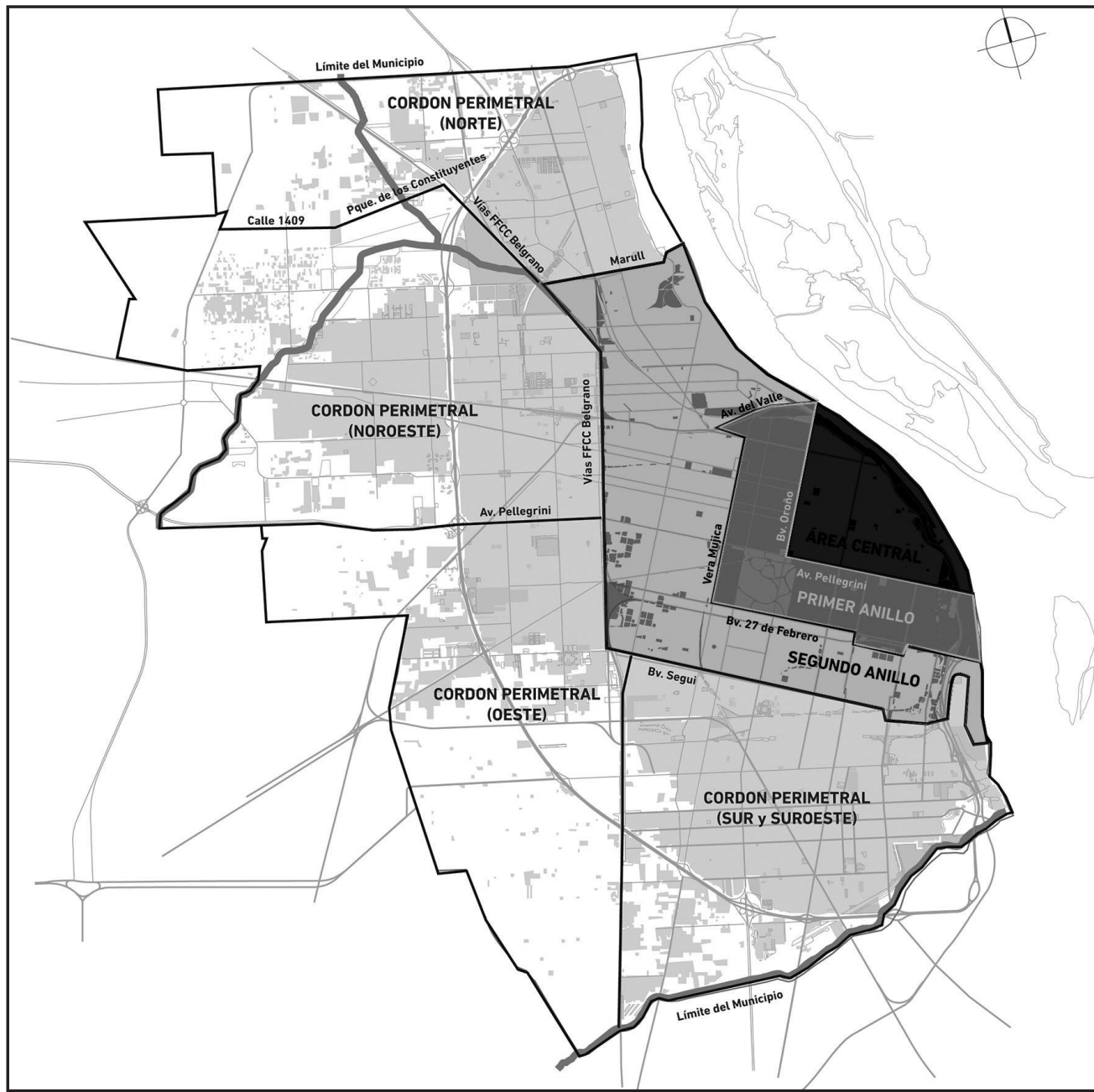

Fuente: Ordenanzas 8243, 8244, 8980 y 9068, Municipalidad de Rosario. 
las administraciones provinciales que redactan y aprueban sus propias constituciones provinciales, por ser la Argentina un país federal. Cada ciudad, por lo tanto, cuenta con la atribución de aprobar o no los proyectos de nuevas edificaciones, según las normas municipales que afectan, limitan y establecen los llamados indicadores urbanísticos.

Los municipios disponen además de un cierto poder tributario (facultad de recaudar o administrar tributos) que también es delegado por la Constitución Nacional y las respectivas constituciones provinciales. Ejercen competencia tributaria sobre la Contribución por Mejoras, las tasas, los derechos y sellos. En relación con estos tributos autorizados, los municipios pueden fijar sus montos, reglamentarlos, gestionar su cobro y administrarlos de acuerdo a las ordenanzas municipales que los rigen (Cuenya et al., 2004).

En el caso de Rosario, es La Ley Provincial Orgánica de las Municipalidades 2756/86 la que le otorga expresamente al municipio, entre otras atribuciones, la facultad de determinar el aprovechamiento edilicio de los terrenos (factor de ocupación e índice de edificabilidad); la altura de los edificios particulares; la línea de edificación, el ancho de las ochavas; la nivelación de las calles de la ciudad y la distancia que deben guardar los propietarios de predios contiguos para construir cercos o paredes medianeras.
En cuanto al poder tributario del Municipio de Rosario, este se encuentra definido por normas de diverso alcance: la Constitución de la Provincia de Santa Fe, diversas leyes orgánicas dictadas por la legislatura provincial (Ley Orgánica de las Municipalidades y Ley de Comunas), y el código tributario municipal. Si bien, estas normas legales no le permiten al municipio crear impuestos, sí aluden a otros tributos como tasas, derechos, contribuciones y otros recursos municipales.

En el marco de ese conjunto de disposiciones legales se definió la Contribución Compensatoria como un aporte en dinero, obras públicas o suelo que los propietarios/urbanizadores deben abonar al municipio por la obtención de la autorización que este les otorga para un mayor aprovechamiento edilicio del suelo.

Además de la semejanza ya señala con la Contribución Onerosa de Brasil (OODC) de Brasil, el instrumento rosarino se asemeja a los aportes especiales (o aportes compensatorios) propuestos en un estudio realizado por un equipo del Centro de Estudios Urbanos y Regionales (CEUR) a solicitud del Municipio de Rosario en 2004. Los aportes especiales o compensatorios fueron sugeridos en ese estudio para permitir la recuperación de plusvalías generadas por una amplia gama de decisiones regulatorias del municipio. Los Aportes Especiales son un tipo de recurso contemplados expresamente como municipales en las constituciones 
provinciales. Son recursos no tributarios que consisten en compensaciones pecuniarias o en especie, que deben pagar por única vez al municipio los propietarios del suelo que obtengan un permiso que los autorice, ya sea a habilitar el suelo al uso urbano, a subdividir el suelo, a otorgarle al suelo un mayor aprovechamiento constructivo o asignarle un uso diferente al habilitado y que implique una mayor rentabilidad del suelo ${ }^{6}$. Dadas ambas semejanzas, es posible inferir que la Contribución Compensatoria fue concebida como un instrumento de recuperación de plusvalías, cuya operatoria y denominación le permite ajustarse al marco legal e institucional y a la práctica urbanística vigente en la ciudad.

\section{EJEMPLO DE APLICACIÓN DE LA CONTRIBUCIÓN COMPENSATORIA POR APROVECHAMIENTO DE MAYOR ALTURA}

Para desarrollar un ejemplo ilustrativo hipotético de aplicación de la Contribución Compensatoria por aprovechamiento de mayor altura se toma como referencia la figura 2.

Supóngase una parcela "P" frentista al corredor "C", ubicada a $150 \mathrm{~m}$ de una plaza en la misma vereda. Como el área del tejido circundante a dicha parcela (color amarillo) permite $15 \mathrm{~m}$ de altura, si el propietario de la parcela $\mathrm{P}$ aspirara a edificar hasta

Cuenya et. al, 2004. los $24 \mathrm{~m}$ permitidos por la nueva ordenanza para Corredores urbanos (color rojo), debería pagar la diferencia hasta llegar a los $24 \mathrm{~m}$. O sea $9 \mathrm{~m}$ que, en este caso, equivalen a 4 pisos de su proyecto.

Cálculo de la Contribución Compensatoria:

1) Cada piso a edificar cuenta con $110 \mathrm{~m}^{2}$ cubiertos.

2) Total a edificar: 4 pisos $\times 110 \mathrm{~m} 2=440$ $\mathrm{m}^{2}$.

3) Valor del $\mathrm{m}^{2}$ en ler. Anillo Perimetral a diciembre de $2013=891 \mathrm{U} \$ \mathrm{D} / \mathrm{m}^{2}$.

4) Valor total $440 \mathrm{~m}^{2} \times 891 \mathrm{U} \$ \mathrm{D} / \mathrm{m}^{2}=\mathrm{U} \$ \mathrm{D}$ 392.040

5) Liquidación de la Contribución (10\%): U\$D 39.204

Ver planilla de liquidación en: http:// www.rosario.gov.ar/tramitesonline/ verArchivo?id=274\&tipo $=$ docTObj 
FIGURA 2: PORCIÓN DEL TEJIDO URBANO DE LA CIUDAD DE ROSARIO

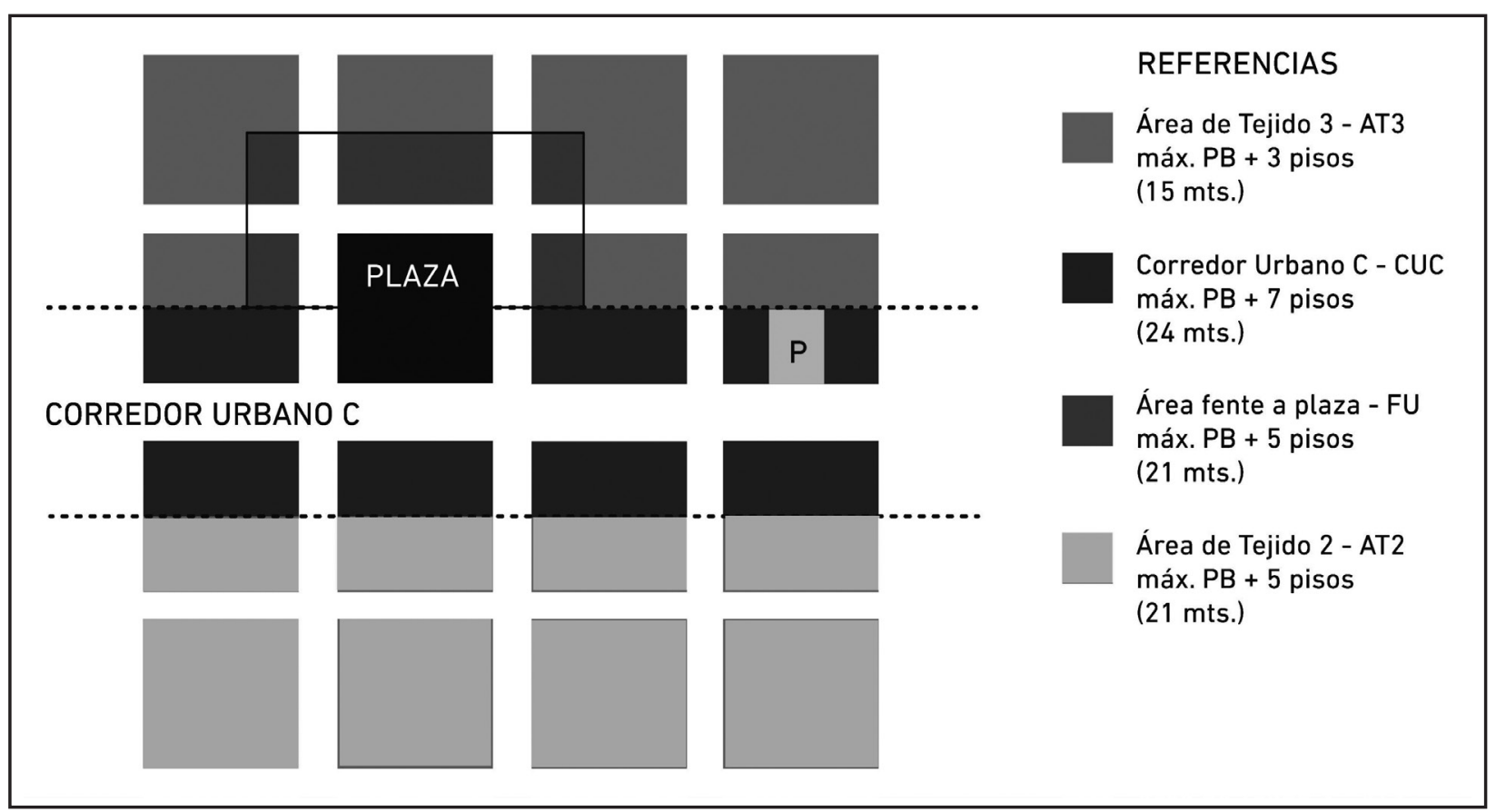

Fuente: Elaboración propia basada en información de la Secretaría de Planeamiento, Municipalidad de Rosario 


\section{ESTIMACIÓN DE LA CONTRIBUCIÓN COMPENSATORIA PARA PROYECTOS EDILICIOS ESPECIALES}

Como se dijo, los proyectos edilicios especiales consisten en operaciones de renovación, rehabilitación y/o sustitución edilicia y se desarrollan en parcelas o grupos de parcelas donde la Municipalidad de Rosario considere pertinente modificar la normativa vigente. A este régimen pueden adherir organismos públicos, actores privados o mixtos (ordenanza 8692/2010).

En estos casos, el monto de la Contribución Compensatoria derivado de los beneficios otorgados en función de la mayor edificabilidad resultante y del mayor aprovechamiento en altura, se obtiene de la aplicación de la siguiente fórmula:

$$
\mathrm{CC}=(15 \% \mathrm{Et}+10 \% \mathrm{~Eb}) \times \mathrm{Vm}^{2}
$$

Donde:

\section{CC: Compensación Compensatoria.}

Et: Edificabilidad en torre (por sobre el basamento).

Eb: Edificabilidad en el basamento por sobre la altura máxima admitida en el tramo.

$\mathrm{Vm}^{2}$ : Valor del metro cuadrado en el mercado.
El Vm² es definido con base en dos (2) tasaciones: una realizada por el Departamento de Tasaciones del Banco Municipal de Rosario y otra, en forma alternada, por los diferentes colegios profesionales cuyos matriculados tengan incumbencias en el tema. Se toma un promedio de las dos tasaciones, si la diferencia entre ambas no supera el 30\% (treinta por ciento) de la correspondiente a la realizada por el Departamento de Tasaciones del Banco Municipal de Rosario. En caso de no ser así, se toma una tercera tasación y el valor considerado es el promedio obtenido entre las tres. Dicho valor se actualiza anualmente de la misma manera, obteniéndose así el valor del metro cuadrado en el mercado, de cada sector afectado por la Ordenanza.

Para la determinación de la compensación económica se computa el 100\% (cien por ciento) de la superficie cubierta involucrada y el 50\% (cincuenta por ciento) de la superficie semicubierta. Los valores de edificación por metro cuadrado establecidos para el cálculo de la compensación económica originada por el mayor aprovechamiento resultante de la ejecución de proyectos especiales, (así como también por mayor aprovechamiento en altura) son anualmente actualizados por el Departamento Ejecutivo y publicados en el Boletín Oficial al momento de su actualización anual. 


\section{EJEMPLO DE APLICACIÓN DE LA CONTRIBUCIÓN COMPENSATORIA PARA EL DESARROLLO DE PROYECTOS DE EDIFICIOS ESPECIALES: EL CASO DEL EDIFICIO RICH}

Se tomó como ejemplo de aplicación efectiva de la Contribución Compensatoria el edificio Rich, ubicado en el área central de la ciudad de Rosario (Calle San Juan nro. 1027/41), puesto que ilustra claramente el potencial que tiene el instrumento para financiar el desarrollo urbano, concretando obras que benefician a la ciudad en su conjunto y a los grupos más desfavorecidos.

El edificio se ubica en una zona de reserva con indicadores urbanísticos aún por definir. El proyecto propuesto por el propietario comprende un restaurante en planta baja, cocheras en planta baja y subsuelos, oficinas en los dos primeros pisos y departamentos residenciales hasta alcanzar los 16 pisos altos. Se desarrolla mediante un basamento, una torre compuesta hasta el piso $11^{\circ} \mathrm{y}$ una torre exenta (de perímetro libre) desde el $12^{\circ}$ al $16^{\circ}$.
El cálculo de la Contribución Compensatoria para este proyecto resultó en 244.000 dólares norteamericanos. El pago se concretó en 305.000 dólares por la actualización anual de los valores de base. Los fondos recaudados se asignaron de la siguiente forma: el 34\% correspondiente al Fondo del Programa Municipal de Construcción de Viviendas para Familias de Ingresos Medios se abonó con dinero en efectivo que se usó para la compra de tierras; el 33\% del Fondo de Preservación se destinó a reparar un centro cultural (Centro Cultural Roberto Fontanarrosa) y la plaza Montenegro que lo contiene, y el restante 33\% del Fondo de Obras Públicas se destinó a financiar parte de las instalaciones del polideportivo perteneciente a una organización vecinal (la Vecinal 7 de Septiembre) en una zona muy pobre de la ciudad y al aporte de materiales para las columnas y luminarias del alumbrado público en un sector de ocho manzanas de la ciudad. 
FIGURA 3. DETALLE DE ESTIMACIÓN DE LA CONTRIBUCIÓN COMPENSATORIA EN EL EDIFICIO RICH

\section{RICH - San Juan 1027/41 \\ Detalle del cálculo para la Compensación Económica}

PLANTA PISO DE $6^{\circ} \mathrm{A} 11^{\circ}$

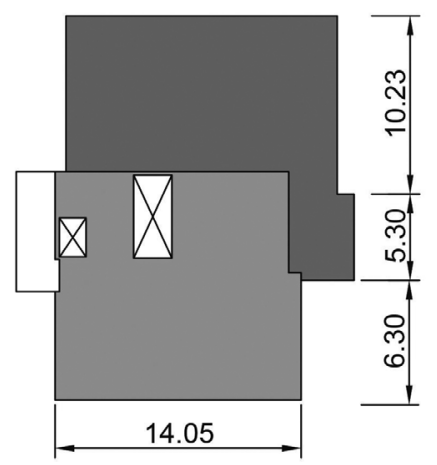

Eb: Edificabilidad en el basamento por sobre la altura máx. admitida en el tramo

$100 \%$ Sup. Cubierta: $161.77 \mathrm{~m} 2 \times 6$ pisos $=970.62 \mathrm{~m} 2$

$100 \%$ Sup. Semicubierta $0.00 \mathrm{~m} 2$

Et: edificabilidad en torre (por sobre el basamento)
PLANTA PISO DE $12^{\circ} \mathrm{A} 15^{\circ}$

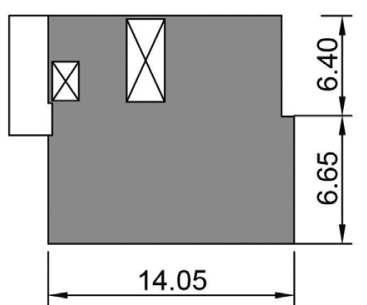

Et: edificabilidad en torre (por sobre el basamento)
PLANTA PISO DE $16^{\circ}$

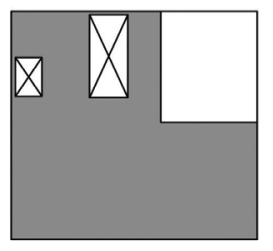

Et: edificabilidad en torre (por sobre el basamento)

Fuente: Elaboración propia en base a expediente municipal nro. 22122-11, Municipalidad de Rosario. 


\section{Desafíos enfrentados durante la implementación de la Contribución Compensatoria}

El proceso de implementación de la Contribución Compensatoria aparejó desafíos en el terreno político, técnico y judicial. En el plano político debió lograrse el consenso necesario para poder aplicar nuevas reglas de juego en cuanto a los derechos y obligaciones asociados a la potencialidad constructiva del suelo urbano y a los beneficios que de allí se derivan a los propietarios. Eso pudo lograrse gracias a un sostenido trabajo de esclarecimiento en el que participaron las cámaras empresariales interesadas y los profesionales y académicos nucleados en la universidad.

Desde el punto de vista técnico, hubo que recalcular los valores por $\mathrm{m}^{2}$ que en las distintas zonas sirven de base al cálculo de la Contribución Compensatoria, ya que la reforma del Código Urbano estableció nuevas potencialidades edificatorias. Si antes de entrar en vigencia el nuevo código estos valores se establecían en base a áreas homogéneas ya establecidas en cada barrio de la ciudad, el nuevo código requirió a los tasadores un análisis pormenorizado por tramos (preservación, completamiento, renovación) dentro de cada nueva área. Se trató de un desafío tanto para el sector público como para los agentes del mercado inmobiliario, que debieron afinar sus metodologías e instrumentos. De hecho, una cámara del sector empezó a dar cursos a tasadores, lo que quedó reflejado en artículo periodístico que con ironía tituló: El nuevo código mandó a la escuela a los agentes inmobiliarios. Un tercer desafío fue tener que afrontar y sortear los reclamos y juicios entablados por los propietarios de inmuebles catalogados como de valor patrimonial, en las zonas donde se redujo la altura de edificación. Esto exigió al municipio argumentar con bases sólidas los criterios preservacionistas. Porque, como reconocen los funcionarios municipales, si bien existen muchos edificios emblemáticos cuyos valores patrimoniales están a la vista, hay muchos otros inmuebles en lo que esto no ocurre. De hecho se plantearon casos de propietarios que dejaron que sus edificios llegaran a la ruina para solicitar al municipio la "descatalogación" como patrimonio y poder construir una torre con rentabilidad asegurada. El municipio perdió algunos juicios. Pero, luego de un trabajo de varios años que convocó a especialistas locales y otros expertos internacionales, se logró armar un inventario patrimonial, que incluye alrededor de 2500 inmuebles -con protección directa- que barre toda la ciudad. Este inventario integra el conjunto de normas del nuevo Código Urbano de Rosario. 
TABLA 1. RECURSOS CAPTADOS EN CONCEPTO DE CONTRIBUCIÓN COMPENSATORIA EN PROYECTOS EDILICIOS REGULARES Y ASIGNADOS A DISTINTOS FONDOS DE DESARROLLO URBANO, 2004-2013

\begin{tabular}{lll}
\hline \multirow{2}{*}{ Fondos de desarrollo urbano } & & Recursos captados \\
\cline { 2 - 3 } & USD $\$$ & $\%$ \\
\hline Fondo de tierras & 14.242 .300 & 58 \\
\hline Fondo de Preservación Urbanística & 4.817 .031 & 20 \\
\hline Fondo Viviendas Clase Media & 607.508 & 2 \\
\hline Fondo de Obras Públicas & 4.817 .031 & 20 \\
\hline TOTAL & 24.483 .870 & 100 \\
\hline
\end{tabular}

Fuente: Informe preliminar sobre convenios urbanísticos, 2013.

\section{Resultados de la aplicación de la Contribución Compensatoria}

De acuerdo a un balance realizado a fines de septiembre de 2013 por la Secretaría de Planeamiento del municipio, se han logrado resultados favorables en varios sentidos.

En primer lugar, entre 2004 y 2013, se firmaron 240 convenios correspondientes a proyectos edilicios regulares en distintas áreas de la ciudad (área centro, áreas de completamiento, corredores urbanos, primer anillo perimetral) y a grandes proyectos urbanos. A través de éstos se recaudaron poco más de 80 millones de dólares norteamericanos. En relación con el exiguo presupuesto municipal ejecutado en 2012 (que ascendía a los 548 millones de dólares norteamericanos) los fondos captados por la Contribución Compensatoria representaron una proporción significativa del casi un $15 \%$ (ver tablas 1 y 2 ). 


\section{TABLA 2. RECURSOS CAPTADOS EN CONCEPTO DE CONTRIBUCIÓN COMPENSATORIA EN GRANDES PROYECTOS URBANOS Y ASIGNADOS A EJECUCIÓN DE ESPACIOS PÚBLICOS, 2004- 2013}

\begin{tabular}{llc|}
\hline \multirow{2}{*}{ Grandes proyectos urbanos } & & Recursos captados \\
\cline { 2 - 3 } & USD $\$$ & $\%$ \\
\hline Puerto Norte & 44.055 .411 & 77 \\
\hline Parque Habitacional Ludueña & 8.585 .797 & 15 \\
\hline Plan Wilde y Newbery & 1.759 .101 & 3 \\
\hline Plan Especial Av. de las 3 Vías & 2.291 .377 & 4 \\
\hline Plataformas Productivas & 659.037 & 1 \\
\hline TOTAL & 57.350 .723 & 100 \\
\hline
\end{tabular}

Fuente: Informe preliminar sobre convenios urbanísticos, 2013.

En segundo lugar, se registró una evolución progresiva de los aportes captados a través de la Contribución, como se observa en la figura 4. Esto da cuenta de la aceptación del instrumento por parte de los desarrolladores, en el marco de una situación favorable del mercado inmobiliario.
Finalmente, se ha valorado como un resultado positivo el hecho de que el grueso de los fondos recaudados, aportados por el cobro de Contribuciones en grandes proyectos urbanos, se asignó a la ejecución de espacios públicos. En el caso de proyectos edilicios regulares, la mayor parte de los recursos captados se asignaron al fondo de tierra. 
FIGURA 4. APORTE DE LA CONTRIBUCIÓN COMPENSATORIA AL FONDO DE PRESERVACIÓN URBANÍSTICA

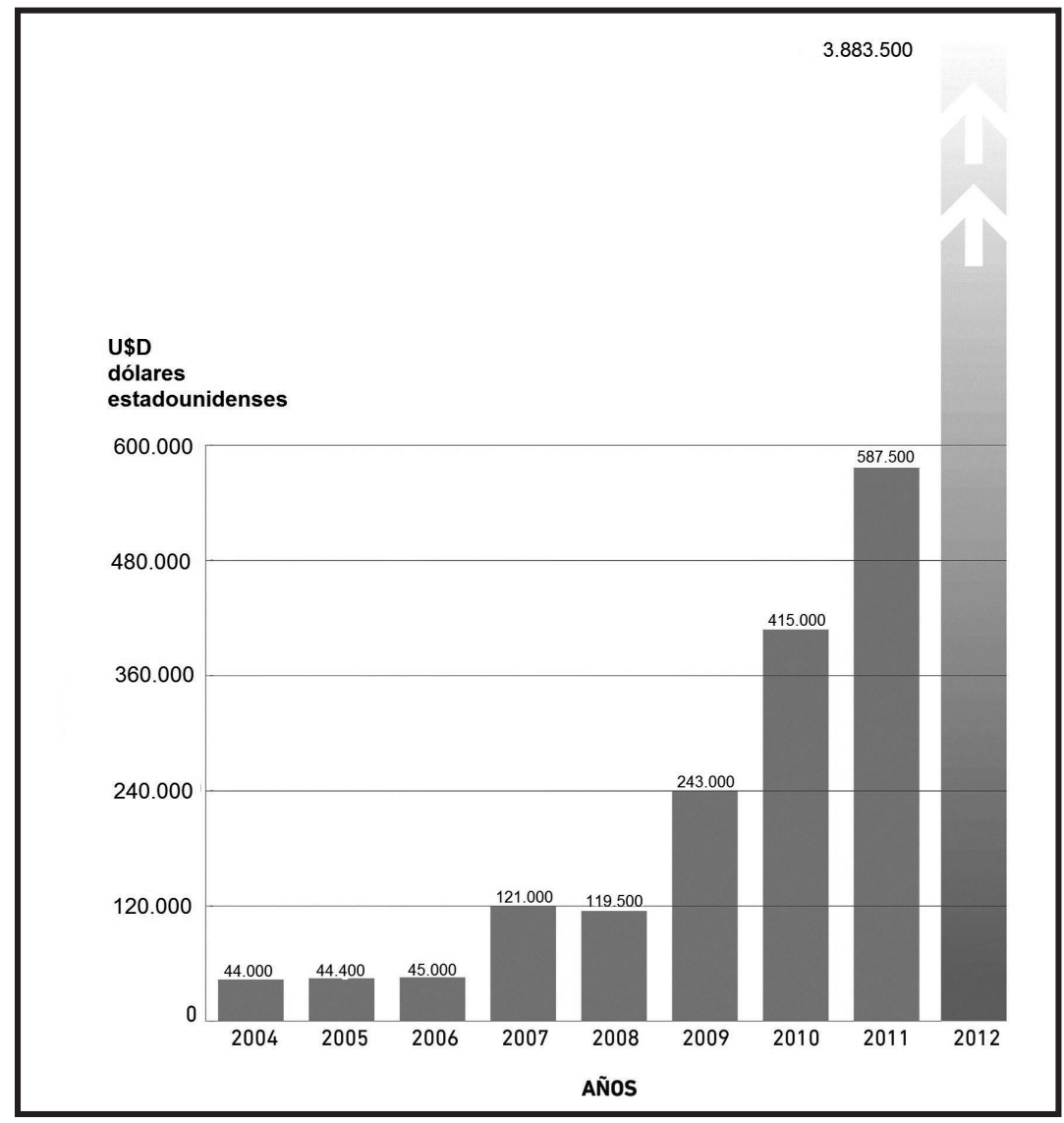

Fuente: Elaboración propia basada en Informe preliminar sobre convenios urbanísticos, 2013. 


\section{Potencial de replicación}

La Contribución Compensatoria es potencialmente replicable en todas las ciudades de la República Argentina, a partir de un ejercicio creativo por parte de los gobiernos locales de las competencias que les son asignadas a los municipios argentinos por la organización federal constitucional.

La reforma constitucional que se instrumentó en 1994 consagró el expreso reconocimiento de la autonomía municipal dentro de nuestro régimen federal, con el alcance y contenido que determinen las provincias, a través de sus constituciones, en el orden institucional, político, administrativo, económico y financiero (artículos 5 y 123). No todas las provincias han armonizado sus marcos constitucionales según el nuevo mandato de la Constitución Nacional. En consecuencia, luego de la reforma, la autonomía municipal va desde un "mínimum" que puede abarcar el establecimiento de sus propias normas de organización, además del autogobierno y la posibilidad de establecer impuestos, hasta un "máximum" de autonomía o "autonomía plena" que supone la capacidad de dictarse la propia Carta Orgánica municipal (Cuenya, et al., 2004).

La provincia de Santa Fe, de la que forma parte el Municipio de Rosario, se encuentra dentro del grupo de provincias que no reconoce la autonomía institucional plena de sus municipios. Por ello, los municipios no pueden dictar su propia norma fundamental para organizar su régimen local y son las normas provinciales las que constituyen el marco para la sanción de sus ordenanzas. Sin embargo, aún con la autonomía institucional limitada, Rosario ha podido crear, aplicar y administrar con un sentido redistributivo la Contribución Compensatoria, presentada en este trabajo.

En consecuencia, si el municipio de Rosario ha logrado aplicar la Contribución Compensatoria en un contexto jurídico restrictivo, es esperable que esta también pueda aplicarse en otras provincias, cuyas constituciones otorgan un mayor grado de autonomía institucional a sus municipios, tales como: Catamarca, Córdoba, Corrientes, Chaco, Chubut, Formosa, Jujuy, La Rioja, Misiones, Neuquén, Río Negro, Salta, San Juan, San Luis, Santa Cruz y Tierra del Fuego. Provincias como Buenos Aires, Entre Ríos y Mendoza aunque no reconocen a sus municipios autonomía institucional plena, les reconocen la facultad para establecer impuestos en la medida dispuesta por la norma general que los rige: la ley orgánica de municipios respectiva.

En cualquier caso, se requiere una clara conciencia por parte de las autoridades (ejecutivas y legislativas) sobre dos cuestiones claves. La primera, es la repercusión del planeamiento urbanístico y la calificación de suelos en los valores inmobiliarios. La segunda alude a la necesidad de operar sobre estos efectos mediante instrumentos fiscales 
o regulatorios que permitan redistribuir las plusvalías urbanas en provecho de la comunidad en su conjunto.

\section{Balance a modo de conclusión}

La aplicación de la Contribución Compensatoria en la ciudad de Rosario es relativamente reciente, por lo cual, todavía resulta escasa la evaluación sobre su alcance y desempeño. No obstante eso, algunos trabajos realizados por autores rosarinos destacan más sus méritos que sus limitaciones.

El trabajo de Bragos y Mazzaro (2012) considera que la política puesta en marcha en Rosario con este instrumento se orienta por principios de equidad y cohesión territorial. Sostiene que su aplicación ha permitido generar un número significativo de proyectos de nueva edificación y de preservación patrimonial. Evalúa que fueron muy significativos los recursos recaudados en las grandes operaciones urbanas, como Puerto Norte y parque habitacional Ludueña. En el primer caso, la Contribución Compensación implicó un cambio en la lógica de actuación del municipio: a diferencia de lo que ocurría en el pasado, cuando era siempre el Estado el que contribuía a la valorización de los inmuebles estimulando la renovación edilicia y dando lugar a buenos negocios inmobiliarios, con el nuevo instrumento,

"la construcción del espacio público queda a cargo de los emprendedores, quienes además, deben donar la tierra necesaria para esos nuevos espacios públicos. De esta manera, la ciudad gana nuevos parques y paseos y la Municipalidad continua con su proyecto de garantizar el acceso público a la costa y de transformar el borde sobre el rio en un gran parque polifuncional" (Bragos y Mazzaro, 2012, p. 19).

La objeción que se hace se refiere a que el destino de los fondos (espacio público dentro del perímetro) agrega valor al proyecto de los emprendedores, cuando lo deseable sería que "tuvieran como finalidad distribuir los beneficios de la actividad inmobiliaria de un modo más equitativo. O sea, derivando estos ingresos hacia los sectores más carentes de la ciudad" (Bragos y Mazzaro, 2012, p. 20). Se reconoce, sin embargo, que la gestión de Puerto Norte también ha demandado la donación de tierras para construir viviendas sociales. El proyecto habitacional Ludueña, en cambio, parece responder según los autores a ese enfoque de mayor redistribución geográfica de los beneficios. Aquí, parte de las obligaciones a cargo del emprendedor consisten en construir espacio público en una de las zonas más marginales de la ciudad, en el Distrito Oeste.

Un segundo trabajo al que se tuvo acceso es la presentación realizada por el equipo de profesionales del Departamento de Registro y Tasación de la 
Dirección General de Tramitación y Fiscalización Urbanística de la Municipalidad de Rosario, en la que, bajo el título "Recuperación de la valorización de la tierra. El caso de la ciudad de Rosario" se exponen los fundamentos y procedimientos para el cálculo de las compensaciones en tres proyectos emblemáticos: Parque Habitacional Ludueña, Parque Norte y Casino Rosario. El trabajo concluye resaltando una frase del tasador en jefe de dicho Departamento, el Ing. Enrique Bosch, quien sostiene lo siguiente:

"La aceptación por parte de los inversores y desarrolladores de abonar el impuesto o compensación por las plusvalías obtenidas para ser empleadas por la administración municipal en obras a favor de la comunidad constituye un cambio en el pensamiento social de los mismos y un logro trascendente de los gobiernos locales" (Álvarez, s.f.).

El trabajo de Peinado, Barenboim, Lagarrigue y Nicastro (2015) es más crítico que los anteriores. Aunque valora la cantidad de obras públicas, vivienda social y equipamiento realizados con recursos municipales limitados, considera que

"la inversión privada total motorizada a través de estos convenios urbanísticos apenas supera el gasto de la Secretaría de Obras Públicas municipal en el período fiscal 2013, y a su vez representa un 15,6\% de los recursos de origen municipal en dicho periodo fiscal" (Peinado et al., 2015, p. 36).

También se cuestiona que parte de los recursos captados bajo la forma de dinero en efectivo se usaron para financiar gastos generales del municipio, en lugar de canalizarse hacia el Fondo de tierras, debilitando el impacto redistributivo de la herramienta.

Desde nuestro punto de vista, la Contribución Compensatoria, aún con sus limitaciones, es un instrumento notable que expande la posibilidad de acción del gobierno local sobre el control del suelo, a través del ejercicio de un planeamiento urbano potente e inductor: un planeamiento que junto con el reconocimiento del derecho de construir (intrínseco a la propiedad privada del suelo) establece límites y obligaciones a ese derecho, con lo cual, logra fijar límites a los irrestrictos derechos de propiedad privada del suelo en representación del interés general. La implícita separación entre derecho de propiedad del suelo y derecho de construir le permite al sector público recuperar (y redistribuir) los incrementos en el valor del suelo que resultan del desarrollo de derechos, más allá de una determinada línea de base establecida en el plan.

Eso posibilita una redistribución más justa de oportunidades y costos del desarrollo urbano, entre el sector público, los propietarios y desarrolladores y la sociedad en general.

Sin contar con un marco normativo general que otorgue prioridad a la función social de la propiedad (como sí lo tiene la Contribución Onerosa brasileña) la Contribución Compensatoria de Rosario 
logra sortear la tradicional idea de que los derechos privados de propiedad anulan el interés público. Se abona así la idea de que es posible contribuir a una ciudad más justa dentro de la democracia política y a través de instrumentos renovados de planificación urbana.

\section{Bibliografía}

Alfonsín, B. d. M. (2008). A politica urbana em disputa: desafios para a efetividade de novos instrumentos em uma perspectiva analítica de direito urbanístico comparado (Brasil, Colômbia e Espanha). (Doutorado), Universidade Federal do Rio de Janeiro Rio de Janeiro. Recuperado de: http://www.ippur.ufrj.br/ download/pub/BetaniaDeMoraesAlfonsin.pdf

Álvarez, R. (s.f.). Recuperación de la valorización de la tierra. El caso de la ciudad de Rosario. Rosario: Secretaría de Planeamiento, Municipalidad de Rosario.

Bragos, 0. y Mazzaro, P. (2012). Desarrollo urbano, equidad territorial e instrumentos de recuperación de plusvalias. Proyectos y realizaciones. Rosario: Facultad de Arquitectura, Planeamiento y Diseño, Universidad Nacional de Rosario.

Cuenya, B., González, E., Mosto, G. y Pupareli, S. (2012). Movilización de plusvalías urbanas en un gran proyecto urbano. El caso de Puerto Norte, Rosario. En: B. Cuenya, P. Novais y C. Vainer (Eds.), Grandes proyectos urbanos. Miradas críticas sobre la experiencia argentina y brasileña (pp. 67-118). Buenos Aires: Café de las Ciudades.

Cuenya, B., Pupareli, S., Brunstein, F., Cascella, H., Mosto, G., Di Loreto, M., Garat, P. y Smolka, M. (2004). Análisis y proposición de métodos de ponderación y de instrumentos de recuperación de plusvalias urbanas generadas por la acción pública municipal. Informe final de consultoría. Buenos Aires: Centro de Estudios Urbanos y Regionales.

Fernandes, E. (2006). A nova ordem jurídico-urbanística no Brasil. En: E. Fernandes y B. Alfonsin (Eds.), Direito urbanístico: estudos brasileiros e internacionais (pp. 3-25). Belo Horizonte: Del Rey.

Furtado, F. (1997). Instrumentos para la recuperación de plusvalias en América Latina. Lincoln Institute of Land Policy.

Jaramillo, S. (2001). La experiencia colombiana en la recuperación estatal de los incrementos del precio del suelo. La contribución de valorización y la participación en plusvalías. En: M. Smolka y F. Furtado (Eds.), Recuperación de plusvalías en América Latina. Alternativas para el desarrollo urbano (pp. 71-98). Chile: Pontificia Universidad Católica de Chile, Linconl Institute of Land Policy.

Levin, M. (2011). Presentación. Plan urbano Rosario 20072017. Secretaría de Planeamiento. Rosario.

Peinado, G., Barenboim, C., Lagarrigue, P. y Nicastro, M. (2015). Los convenios urbanisticos en la ciudad de Rosario, Argentina, como instrumentos de gestión del suelo. Análisis de sus impactos en términos de equidad. Lincoln Institute of Land Policy. 
Rolnik, R. (2001). Estatuto da cidade. Instrumento para as cidades que sonham crescer com justicia e beleza. En: N. Saule Junior y R. Rolnik (Eds.), Estatuto da cidade: novas perspectivas para a reforma urbana (pp. 5-9). Sao Paulo: Polis.

Sandroni, P. H. (2011). Recent experience with land value capture in Sao Paulo, Brasil. Land Lines, 23(3), 14-19.

Secretaría de Planeamiento, Municipalidad de Rosario (2013). Informe preliminar sobre convenios urbanísticos. Rosario: Autor.

Secretaría de Planeamiento, Municipalidad de Rosario (2011). Plan urbano Rosario 2007/2017. Rosario: Autor.

Smolka, M. (2013). Implementig value capture in Latin America. Policies and tools for urban development. Cambridge, MA: Lincoln Institute of Land Policy.

Smolka, M. y Furtado, F. (2014). Instrumentos notables de politicas de suelo en América Latina. Ecuador: Lincoln Institute of Land Policiy, Ministerio das Cidades, Banco del Ecuador. 